\title{
An efficient similarity indexing by ordering permutations for Spatial Multi-Resolution images
}

\author{
Rachid Alaoui, Said Ouatik El Alaoui and Mohammed Meknassi
}

\begin{abstract}
Color histogram is one of the common techniques used in image retrieval systems. However, the main problem with color histogram indexing is that it does not take the color spatial distribution into consideration. In this paper, we introduce the local histograms to describe the spatial information of colors, even though, a single local histograms is not enough for efficient and robust image retrieval system. We propose the use of color local histograms on multiresolution images. The multiresolution color local histograms give much better retrieval efficiency. The multiresolution images are generated using the median filter. to measure similarity between two images using the local histograms, the traditional approaches use a metric distance. To improve performance of the CBIR system, the non metric measure (PMM) is used to measure similarity of images instead of classic metric. Experiment results prove that the CBIR using our new measure has better performance and perceptually relevant result.
\end{abstract}

Index Terms - color multiresolution image; histogram; Indexing; Similarity measure.

\section{INTRODUCTION}

Recently, the development of image/video archives grows rapidly due to the advancement and popularization of internet technologies and high-capacity data storage devices. Among them, image retrieval plays an important role because this kind of system can help people efficiently find those images which they want to browse in the database. Right now, research on content-based image indexing and retrieval is mainly focused on the visual features of color, texture and shape. When building an image database, feature vectors from images are to be extracted and then store the vectors in another database for future use. When given a query image its feature vectors are computed. If the distance between feature vectors of the query image and image in the database is small enough, the corresponding image in the database is to be considered as a match to the query. The search is usually based on similarity rather than on exact match and the retrieval results are then ranked accordingly to a similarity index. The block diagram of basic CBIR (Content Based Image Retrieval) system is as shown in Fig. 1.

Manuscript received March 28, 2009.

Rachid Alaoui, Said Ouatik El Alaoui and Mohammed Meknassi Laboratory LISQ, Department of Mathematics and Informatics at the Faculty of Sciences Dhar-Mahraz Fes, Morocco. Phone: (212) 662629653.

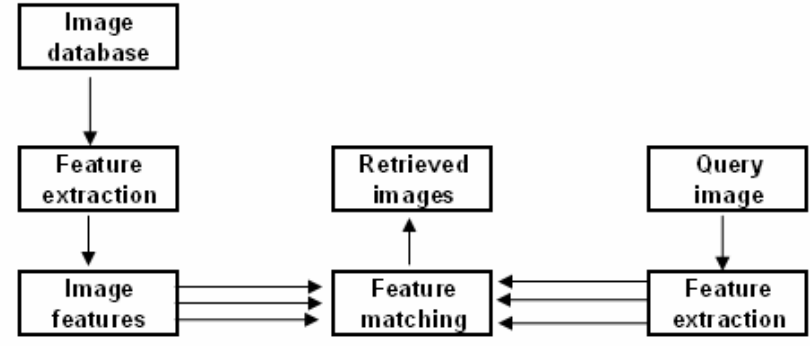

Fig. 1: Block diagram of CBIR

Many color-related features can be extracted, such as dominant colors, color distributions, color layout, etc. Among them, color histogram is the most commonly used technology in color-based image retrieval systems. Color histogram describes the overall color distributions of an image, i.e., it describes what colors are in an image and in what quantities. The use of color histogram for image retrieval has been widely explored by Swain and Ballard [1].

For simplicity, let's I denote a digital image and $|I|_{\text {denote }}$ the size of the image. Then, we discretize its color space into m distinct colors $c_{1}, \ldots, c_{m}$. Finally, a normalized histogram is computed by dividing the frequency of each color bin by the size of the image. Therefore, the normalized histogram of image $H_{I}$ can be defined as:

$H_{I}\left(c_{i}\right)=\frac{\sum_{x} \sum_{y} \operatorname{color}\left(I_{x, y}\right)=c_{i}}{|I|}$

A main advantage of using a histogram is its robustness with respect to the projection of the image. Color histograms are invariant to translation, rotation around the viewing axis, and change slowly with distance to the object and partial occlusion. However, the histogram captures only the color distribution in an image and does not include any spatial correlation between individual pixels. Such indexing can potentially give false results on image queries. Sometimes, two images with dramatically different semantics can give rise to similar histograms. To reduce the problem, several schemes including spatial information have been developed. Recently, several schemes including spatial information have been proposed. Color correlogram [2] and color coherence vector [3] can combine the spatial correlation of color regions as well as the global distribution of local spatial correlation of colors. These techniques perform better than 
traditional color histograms. However, they require very expensive compotation. Histograms of local appearance descriptors (Boujemaa et al., 2001) [4] are a popular representation for visual recognition. They are highly discriminate and they have good resistance to local occlusions and to geometric and photometric variations.

Finding the similarity between local histograms vector is an important field in content. Therefore, a number of measures for calculating a distance between two local histograms have been proposed. Almost all research work in the Content-Based Image Retrieval (CBIR) community has used a metric function to measure similarity between images.

A metric space is a pair $(X, d)$ where $X$ represents the universe of valid objects and $d: X \times X \mapsto I R^{+}$is a function of object pairs, that returns non-negative real values (the distance between objects in the space) that hold the properties of a metric (strict positiveness, symmetry, and the triangle inequality).

$$
\begin{aligned}
& d(x, y)=0 \quad \Leftrightarrow \quad x=y \\
& d(x, y)=d(y, x) \\
& d(x, y) \leq d(x, z)+d(z, y)
\end{aligned}
$$

Minkowski-like metrics[5] (including Euclidean [4, 6, 7] and Fractional distance functions [8] is a very commonly used distance measurement.

A vector space $R^{d}$ is a particular type of metric space, composed by n-tuples of real numbers called vectors. That is, if $x \in R^{n}$ then $x=\left(x_{1}, \ldots, x_{n}\right), x_{i} \in R^{n}, 1 \leq i \leq n$

A widely used family of distance functions for vector spaces is the Minkowski distance $\left(L_{p}\right)$, which we define as

$$
L_{n}(x, y)=\left(\sum_{i=1}^{n}\left|x_{i}-y_{i}\right|\right)^{1 / n}
$$

Another form of metric indexing is the cosine distance $[9,10]$ it can be calculated with Eq. 6 .

$$
\cos \theta=\frac{\sum_{j=1}^{n} x_{j} y_{j}}{\sqrt{\sum_{j=1}^{n}\left(x_{j}\right)^{2}} \sqrt{\sum_{j=1}^{n}\left(y_{j}\right)^{2}}}
$$

Generally speaking, different distance functions should be used depending on the type of data presented by the application. Using a certain distance measure one can decide if the local histograms are similar or not using the following decision rule: the local histograms are similar if the distance between them is less than some threshold. A permutation of the components of vector $\mathrm{x}$ or $\mathrm{y}$ will change the value of distance because a metric is strictly positive. In many system indexing scenarios, spatial similarity technique cannot be used efficiently by a metric function. We showed that these methods frequently overlook obviously similar images and, hence, are not adequate for recognizing nearreplica images. For example, Boujemaa [4] used a Minkowski distance $\left(L_{p}\right)(\mathrm{p}=1)$ for measuring similarity between two images using the local histograms. However, the distance $L_{1}$ has a serious drawback as follows. It is reasonable that if two images have the same content visual can be considered similar. But in large bases images that measure $L_{1}$ becomes low to catch any deviation of visual content by the transformations: rotation and translation. It's the same thing when working with another distance. Therefore due to the drawback, some results of the image retrieval are not so effective.

In this paper we first present a non-metric distance function, permuto-metric measure (PMM), which works significantly better than Minkowski-like functions for measuring perceptual similarity, However, here we present briefly this procedure to give an idea of the research in which our similarity measure is applied. Using PMM remains more stable performance by transformations geometry and provides a superior retrieval performance compared to the usual distance $L_{1}$. Even though, a single color local histograms of images is not enough for efficient image retrieval, we suggest to use a description spatial on multiresolution images for more efficient image retrieval. Multiresolution images using wavelets [11], Gabor filters [12], Gaussian filters [13] and spatial filters [15] were used before for histograms. We decompose the images into different resolution using simple median filtering of different sizes. The images are filtered till three to five level resolutions. The color local histograms are then calculated for different resolution images.

\section{THE LOCAL HISTOGRAMS DESCRIPTOR}

The problem of the color histogram is that only the color distribution in an image is captured. The local histograms vector (Boujemaa et al., 2001) [4] is an effort to merge the spatial content. Intuitively, the local histogram expresses how the spatial geometry of color in the windows. An image $\mathrm{I}$ is evenly divided into a number of $\mathrm{n}$ non-overlapping windows, and each individual window is abstracted as a unique feature descriptor with its spatial location, suppose $\mathrm{Ai}$ be the set of pixels with color bin $\mathrm{i}$ for $\mathrm{i}=1, \ldots, \mathrm{m}$ of an image I and $h(i)$ be the number of elements in Ai. Let $h_{j}(i)$ be the count of the pixels of color bin $\mathrm{i}$ inside window $\mathrm{j}$. Then the local color histograms can be written as $\left(h_{1}(i), h_{2}(i), \ldots, h_{n}(i)\right)$

Based on the local color histograms, the NLDH (the normalized local distribution histogram) can be defined as $\tilde{h}(i) \quad$ where $\quad \tilde{h}(i)=\left(\tilde{h}_{1}(i), \tilde{h}_{2}(i), \ldots, \tilde{h}_{n}(i)\right)$ and $\tilde{h}_{j}(i)=\frac{\left|h_{j}(i)\right|}{|h(i)|}$

The $L_{1}$ distance between two histograms can be written:

$$
d_{L_{1}}\left(\tilde{h}^{1}, \tilde{h}^{2}\right)=\sum_{i=1}^{n} \sum_{c=1}^{m}\left|f\left(\tilde{h}_{i}^{1}(c)\right)-f\left(\tilde{h}_{i}^{2}(c)\right)\right|
$$

We have a simplification expression for $d_{L_{1}}$ as:

$$
\begin{aligned}
d_{L_{1}}\left(\tilde{h}^{1}, \tilde{h}^{2}\right) & =\sum_{i=1}^{n} d\left(\tilde{h}^{1}{ }_{i}, \tilde{h}^{2}{ }_{i}\right) \\
\text { with } d\left(\tilde{h}^{1}{ }_{i}, \tilde{h}^{2}{ }_{i}\right) & =\sum_{c=1}^{m}\left|f\left(\tilde{h}_{i}{ }^{1}(c)\right)-f\left(\tilde{h}_{i}{ }^{2}(c)\right)\right|
\end{aligned}
$$


To have good results of this distance, one takes $f(x)=x^{\alpha} \quad$ with $0<\alpha<1$ (for our experimental we choose the value $\alpha=1 / 3$ ).

\section{THE PROBLEM AREAS TO USE THE DISTANCE FOR CHARACTERIZING THE SIMILARITY}

A distance function is used to quantify (dis)similarity between two instances (images). Each image can be represented by a n-dimensional feature vector $x=\left(x_{i}\right)_{1 \leq i \leq n}$ where each dimension represents an extracted feature. In the following, we define the feature distance in feature channel i as $\Delta d_{i}=\left|x_{i}-y_{i}\right|$ for each $1 \leq i \leq n$.

Since we normalize the feature values to be in the range of $[0,1]$, the feature distance is also in the range of $[0,1]$. The Minkowski-like metrics between any two images, $\mathrm{x}$ and $\mathrm{y}$, is defined as

$$
D(x, y)=\left(\sum_{i=1}^{n} \Delta d_{i}^{r}\right)^{1 / r}
$$

When we set $\mathrm{r}=1$, the above distance is the $L_{1}$ distance. When we set $r=2$, it is the Euclidean or $L_{2}$ distance. And, when $r$ is a fraction, it defines a fractional function.

In general, a permutation of the components of vector $\mathrm{x}$ or $\mathrm{y}$ will change the value of distance between $\mathrm{x}$ and $\mathrm{y}$ because a metric is strictly positive.

Therefore, mathematically, we have the following condition:

$x$ and $y$ are similarly by $D \Leftrightarrow x_{i}=y_{i}$ for each $1 \leq i \leq n$

Based on this condition, in many system indexing scenarios, D becomes low to catch any deviation of visual content by the transformations geometry.

Indeed, in big bases of images, for example, the $L_{1}$ distance becomes weak to capture all deviation of visual content image by rotation or translation, the figure 2 present two images in black and white (every image is subdivided into square tiles (e.g., $2 \times 2$ ) that have the same general content but they are different by the measure classic $L_{1}$. Indeed

$$
d_{L_{1}}\left(\tilde{h}^{1}, \tilde{h}^{2}\right)=\sum_{i=1}^{4} d\left(\tilde{h}^{1}{ }_{i}, \tilde{h}^{2}{ }_{i}\right) \geq d\left(\tilde{h}_{1}{ }^{1}, \tilde{h}_{1}{ }^{2}\right)(11)
$$

The distance $d\left(\tilde{h}_{1}{ }^{1}, \tilde{h}_{1}{ }^{2}\right)$ is very big because the window 1 in the two images 1 and 2 are different in color.

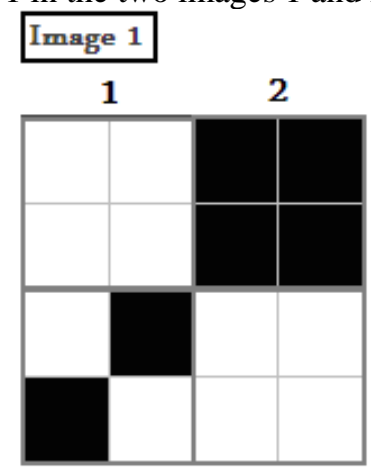

3

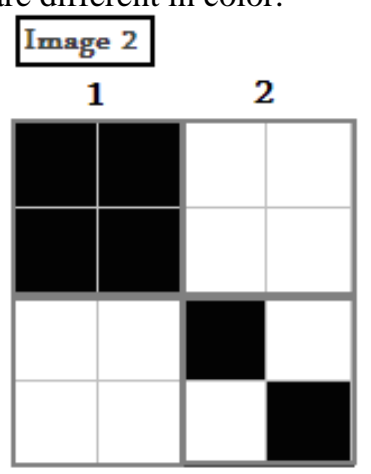

3
Fig. 2: two images with the same visual content, but with different similarity by measure $L_{1}$.

Spatial indexing is a real-life application where similarity searching has to be carried out over a space that is not even metric. The powerful idea in this paper proposes the permuto-metric measure (PPM) to measure similarity between two images, $Z: \Omega^{n} x \Omega^{n} \rightarrow I R^{+}$, satisfying the condition:

$$
D(x, y)=0 \Leftrightarrow x \in \sigma(y) \quad \text { (12) }
$$

$\sigma(y)$ : The set of vectors where its components are the permutations of component of $\mathrm{Y}$. to implement our topological strategy, we are going to characterize this form of application explicitly.

\section{THE PERMUTO-METRIC MEASURE:}

One important issue for researchers of content-based image retrieval systems is finding good models and similarity measures which best mirror human perception for comparing images, and which can be integrated into the systems, in this section, we developed a new measure PMM remains more stable performance by transformations geometry.

We need a bit terminology. Let $x=\left(x_{i}\right)_{1 \leq i \leq n}$ a vector, the set of vectors where its components are the permutations of component of $\mathrm{x}$ is noted $\sigma(x)$ and of cardinal $\mathrm{n}$.

Definition 1. A permuto-metric on the set $\Omega^{n}$ is application $\psi: \Omega^{n} x \Omega^{n} \rightarrow I R^{+}$

who satisfies the axioms according to:

$1-\Psi(x, y)=0 \Leftrightarrow x \in \sigma(y)$

$2-\psi(x, y)=\Psi(y, x)$

$3-\Psi(x, y) \leq \Psi(x, z)+\Psi(z, y)$

One can call $\Psi$ the permuto-metric measure.

The couple $\left(\Omega^{n}, \Psi\right)$ is called a permuto-metric space.

Let's notice that the only difference between the definition and the one of a metric space is the permuted separation replaces the usual separation:

$$
\Psi(x, y)=0 \Leftrightarrow x=y
$$

Therefore, two distinct points can be a null distance in a permuto-metric space.

However, the relation $\Psi^{*}$, definite in by the formula:

$$
x \Psi^{*} y \Leftrightarrow y \in \sigma(x)
$$

is a relation of equivalence, one can consider the class of equivalence of one $\mathrm{x}$ point formed by the set of vectors where its components are the permutations of component of $\mathrm{x}$, therefore:

$$
\overline{x(\psi)}=\left\{y \in \Omega^{n} / y \in \sigma(x)\right\}
$$

Thus, $\overline{x(\Psi)}$ is the closing of $\mathrm{x}$ for the permuto-metric topology. The space quotient of $\Omega^{n} \quad$ under $\Psi^{*}$ is noted by:

$$
\overline{\Omega(\Psi)}=\left\{\overline{x(\Psi)} \quad / \quad x \in \Omega^{n}\right\}
$$

The application $\bar{\Psi}: \overline{\Omega(\Psi)} \times \overline{\Omega(\Psi)} \Rightarrow I R \quad$ defined by $\bar{\Psi}(\bar{x}, \bar{y})=\Psi(x, y)$ is therefore a metrics for the space 
quotient. Let's notice that $\Psi$, in the case or is already a metrics, the space quotient $\overline{\Omega(\Psi)}$ is homeomorphism to $\Omega^{n}$.

Otherwise, a permuto-metric can be treated as the function of a variable that measures the distance to a fixed point.

Definition 2. E a set, let $\tilde{d}: E^{n} \times E^{n} \rightarrow I R^{+}$a distance, $\tilde{d}$ called permuto-symmetrical if

$\forall(x, y) \in E^{n} \times E^{n}, \quad \forall \bar{y} \in \sigma(y), \quad \exists \bar{x} \in \sigma(x)$, for that $\tilde{d}(x, \bar{y})=\tilde{d}(\bar{x}, y)$

Example 1. A Minkowski -distance

$N_{p}(x, y)=\left(\sum_{i}\left|x_{i}-y_{i}\right|^{p}\right)^{1 / p}$ is permuto-symmetrical.

Theorem 1. E a set, let $\tilde{d}: E^{n} \times E^{n} \rightarrow I R^{+}$a distance permuto-symmetrical, then the application: $Z: E^{n} \times E^{n} \rightarrow I R^{+}$defined in the following way: $Z(x, y)=\inf _{s \in \sigma(y)} \tilde{d}(x, s)$ is a permuto-metric measure.

\section{Proof}

Let $(x, y) \in\left(E^{n}\right)^{2}$

(1) $Z(x, y)=0 \Leftrightarrow \quad x \in \sigma(y)$ evident.

(2) Let us show that $\mathrm{Z}(\mathrm{x}, \mathrm{y})=\mathrm{Z}(\mathrm{y}, \mathrm{x}) \forall(x, y) \in\left(E^{n}\right)^{2}$ Indeed, let $(x, y) \in\left(E^{n}\right)^{2}$ with:

$$
\begin{aligned}
& y=\left(y_{i}\right)_{1 \leq i \leq n}, \quad x=\left(x_{i}\right)_{1 \leq i \leq n} \\
& Z(x, y)=\inf _{s \in \sigma(y)} \tilde{d}(x, s)
\end{aligned}
$$

$\sigma(y)$ is a finite set, so

$\exists \bar{y}=\left(\bar{y}_{i}\right)_{1 \leq i \leq n} \in E^{n}, \bar{y} \in \sigma(y), Z(x, y)=\tilde{d}(x, \bar{y})$

$\tilde{d}$ is a distance permuto-symmetrical then

$\exists \bar{x} \in \sigma(x)$ for that $Z(x, y)=\tilde{d}(\bar{x}, y)$

$Z(x, y)=\tilde{d}(y, \bar{x})$ Because $\tilde{d}$ is symmetric

$Z(x, y) \geq Z(y, x)$

Similarly we prove $Z(y, x) \geq Z(x, y)$

So $Z(x, y)=Z(y, x)$

(3) Let us show

that

$Z(Q, P) \leq Z(Q, K)+Z(K, P), \quad \forall(P, Q, K) \in\left(E^{n}\right)^{3}$

With $P=\left(p_{i}\right)_{1 \leq i \leq n}, Q=\left(q_{i}\right)_{1 \leq i \leq n}, K=\left(k_{i}\right)_{1 \leq i \leq n}$

One has:

$$
\begin{aligned}
& Z(Q, P)=\inf _{y \in \sigma(P)} \tilde{d}(q, y) \\
& \leq \tilde{d}(q, \varphi)+\inf _{y \in \sigma(P)} \tilde{d}(\varphi, y) \quad \forall \varphi \in \sigma(K)
\end{aligned}
$$

$$
\begin{aligned}
& \leq \tilde{d}(q, \varphi)+\inf _{x \in \sigma(\varphi)} \tilde{d}(x, p) \\
& \leq \tilde{d}(q, \varphi)+\inf _{x \in \sigma(K)} \tilde{d}(x, p) \\
& \text { Because } \sigma(K)=\sigma(\varphi) \\
& Z(Q, P) \leq \tilde{d}(q, \varphi)+Z(K, P) \quad \forall \varphi \in \sigma(K) \\
& Z(Q, P) \leq \inf _{\varphi \in \sigma(K)} \tilde{d}(q, \varphi)+Z(K, P) \\
& Z(Q, P) \leq Z(Q, K)+Z(K, P)
\end{aligned}
$$

\section{End}

To get a measure of similarity stable by rotation or translation, we can be considered the permuto-metric measure Z. $\quad Z(x, y)=\inf _{s \in \sigma(y)} \tilde{d}(x, s) \quad$ with $\tilde{d}(x, y)=\sum_{j=1}^{n} d\left(x_{j}, y_{j}\right)$

Our objective is working out an algorithm for similarity calculation whose result is statistically close to the expert decision. for tested our new measure, two algorithms are developed for constructing $\mathrm{Z}$ :

Algorithm 1 gives the complete pseudocode for constructing $\sigma(x)$ the set of permutations possible for the vector $\mathrm{x}=\mathrm{tab}[\mathrm{]}$

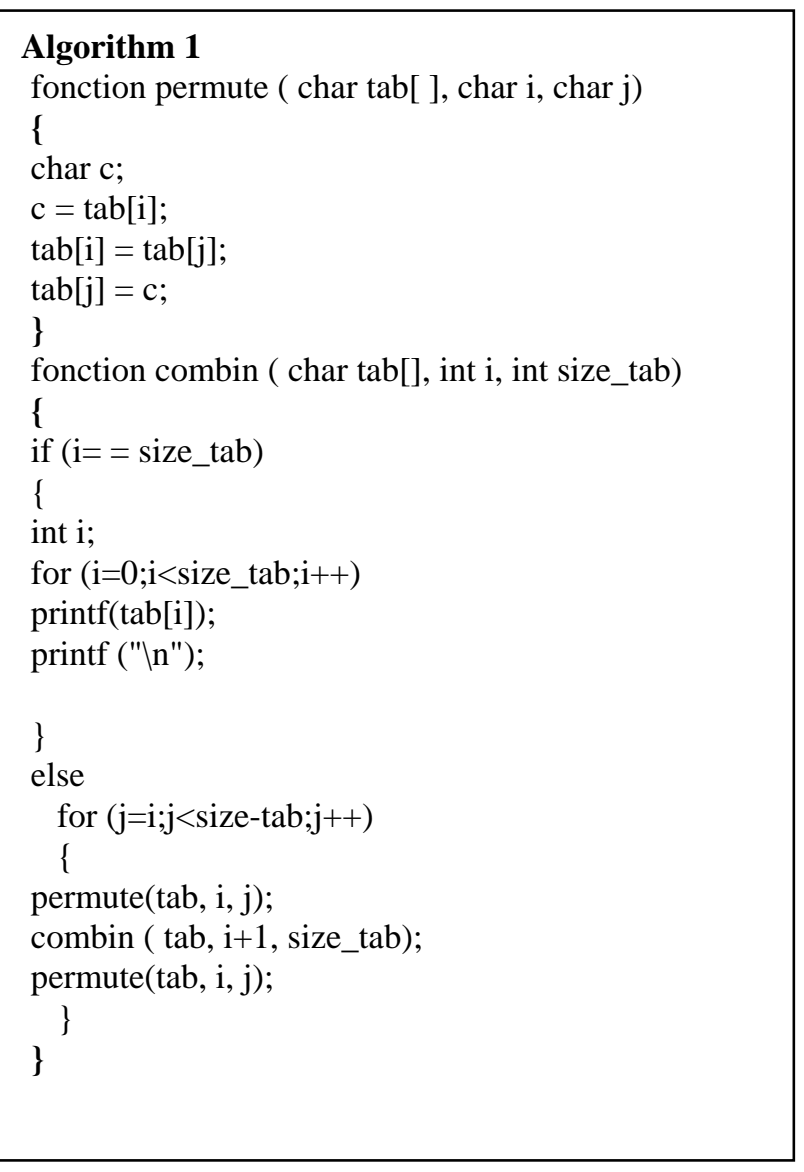

Algorithm 2 provides the details strategy of our system indexing by the measure PMM 


\section{Algorithm 2}

1- find the NLDH vector of query image $\left(I_{q}\right)$,

$w_{Q}=\left(\tilde{h}_{j}^{Q}\right)_{1 \leq j \leq n}$

2- For each $I_{k} \in S$ do

find the NLDH vector of image $\left(I_{k}\right), w_{k}=\left(\tilde{h}_{j}^{k}\right)_{1 \leq j \leq n}$ constructing the set $\sigma\left(\left(\tilde{h}_{j}^{k}\right)_{1 \leq j \leq n}\right)$ using the algorithm 1 calculate the minimum of $\sum_{j=1}^{n} d\left(\tilde{h}_{j}^{Q}, s_{j}\right)$ for

$\left(s_{j}\right)_{1 \leq j \leq n} \in \sigma\left(\left(\tilde{h}_{j}^{k}\right)_{1 \leq j \leq n}\right)$

end for

3- return the top $\mathrm{K}$ images by sorting the similarity measure values $\mathrm{Z}$ in descending order

It is easy to verify that the two images presented in Figure 2 are similar by $\mathrm{Z}$

Indeed

$$
\begin{aligned}
& z\left(\tilde{h}^{1}, \tilde{h}^{2}\right)=\inf _{y \in \sigma\left(\tilde{h}^{2}\right)} \sum_{j=1}^{n} d\left(\tilde{h}_{j}^{1}, y_{j}\right) \\
& =d\left(\tilde{h}_{1}^{1}, \tilde{h}^{2}{ }_{2}\right)+d\left(\tilde{h}_{2}^{1}, \tilde{h}^{2}{ }_{1}\right)+d\left(\tilde{h}_{3}^{1}, \tilde{h}_{4}^{2}\right)+d\left(\tilde{h}_{4}^{1}, \tilde{h}_{3}^{2}\right) \\
& =0
\end{aligned}
$$

\section{IMPROVEMENT}

It is quite difficult to construct a good retrieval system. Most of the systems are based on features that are semantically too primitive. The local histograms descriptor is not enough for efficient image retrieval from a large number of images. An aggregation process is implemented to construct a multiresolution local histograms corresponding to the multiresolution color images. Our new descriptor give better result as local histograms for different resolutions of an image are different, and hence represents different features of images. The histogram of a part (6x6) of an input image quantized to four levels and its lower resolution are different as shown in Fig.3. In Fig.3, we propose to use the multiresolution local histograms instead of single local histograms for efficient image retrieval. Multiresolution images are obtained using the median filtering.

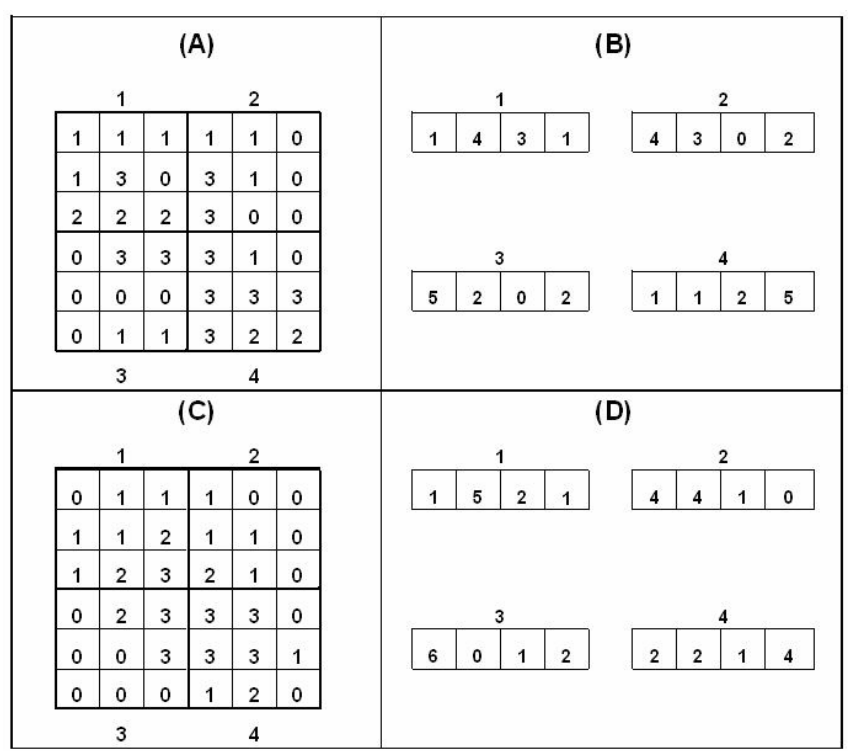

Fig. 3: (A) A portion of an input image for 4 levels color. (B) The local histograms of an input image for 4 windows. (C) The image after $3 \times 3$ median filtering. (D) The local histograms of the image after a median filtering.

Suppose $\mathrm{d}$ be the number of multiresolution levels, $A_{i}^{k}$ be the set of pixels of an image I with color bin i at $k^{\text {th }}$ level and $h^{k}(i)$ be the number of elements in $A_{i}^{k}$. Let $h_{j}^{k}(i)$ be the count of the pixels of color bin $\mathrm{i}$ inside window $\mathrm{j}$ at $k^{\text {th }}$ level. Then the multiresolution local color histograms can be written as $\left(h_{j}^{k}(i)\right)_{1 \leq j \leq n}^{1 \leq k \leq d}$

Based on the multiresolution local color histograms, the NMLH (the normalized multiresolution local histograms) can be defined as $M h$ where

$$
\operatorname{Mh}(I)=\left(\tilde{h}_{j}^{k}(i)\right)_{1 \leq j \leq n}^{1 \leq k \leq d} \text { and } \tilde{h}_{j}^{k}(i)=\frac{h_{j}^{k}(i)}{h^{k}(i) \times d}
$$

The multiresolution images are obtained by passing the original image through the median filters of different sizes. The sizes of median filters for different levels of resolution are selected as:

For $j=1, L 1=1 \times 1 ; j=2, L 2=3 \times 3 ; j=3, L 3=7 \times 7$; $\mathrm{j}=4, \mathrm{~L} 4=11 \times 11 ; \mathrm{j}=5, \mathrm{~L} 5=15 \times 15$ where $\mathrm{Lj}$ represents the size of median filtering, and hence the level of resolution.

It is important to empirically evaluate the effect of different resolution levels, we adopt the following procedure. We give more weights to the higher resolution image local histograms as they contain more information than the lower resolution images. The weight $w_{k}=1 / 2^{k}, 1 \leq k \leq d$ evaluate the effect of different resolution levels.

The measure of similarity tries to capture the strength of relationships between features during comparisons of images in a database. Based on the definition of PMM and NMLH matrix, we propose W-PMM (weighted measure PMM):

$$
\left.\left.\operatorname{MZ}\left(\tilde{h}_{j}^{k}\right)_{1 \leq j \leq n}^{1 \leq k \leq d},\left(\tilde{h}_{j}^{k}{ }^{2}\right)_{1 \leq j \leq n}^{1 \leq k \leq d}\right)=\sum_{k=1}^{d} w_{k} \times Z\left(\tilde{h}_{j}^{k}\right)_{1 \leq j \leq n},\left(\tilde{h}_{j}^{k}{ }^{2}\right)_{1 \leq j \leq n}\right)
$$


Algorithm 3 provides the details strategy of our system indexing by the weighted measure PMM for NMLH matrix

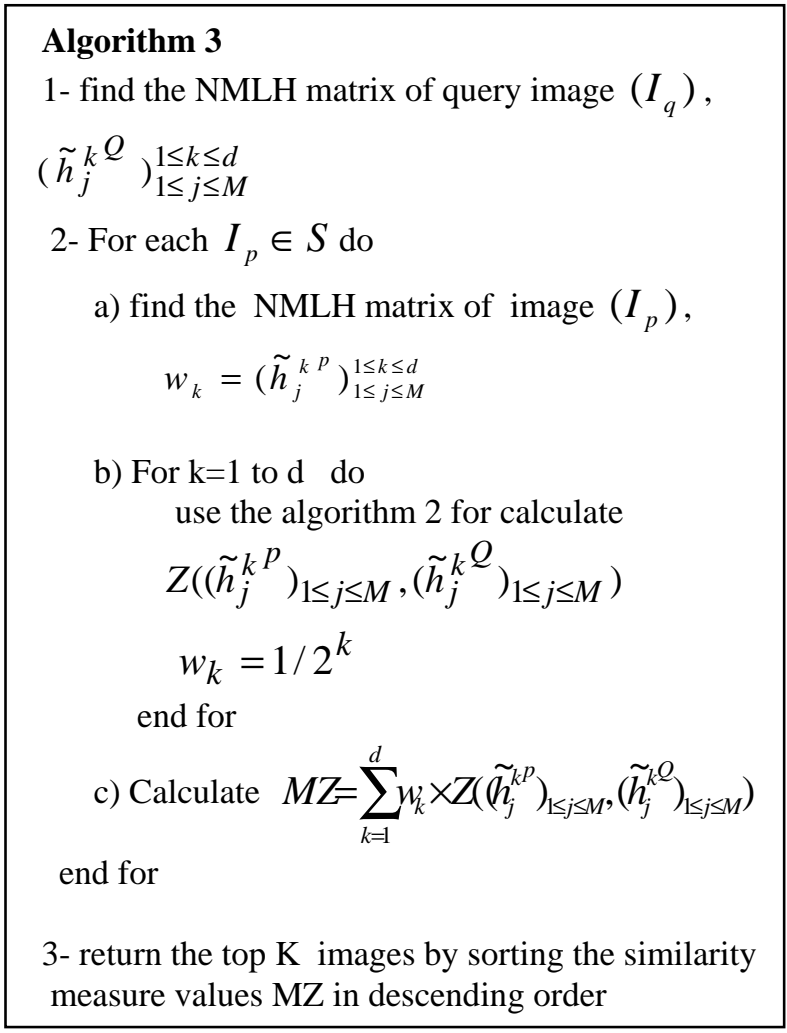

\section{EXPERIMENTAL RESULTS}

Color is one of the most salient features in an image. It is relatively robust to background complication and independent of image size and orientation. The RGB color space is one of the most popular and widely used color spaces in that its format is the most common one for digital images and is compatible to computer display. For simplicity, and as an initial study on this issue, only the RGB color space will be considered. Again, for simplicity of the experiment, color characteristic is not a factor in the study, and the basic uniform partitioning method is chosen. The color space is uniformly quantized into 8 bins / component quantization. Experimental results are illustrated to demonstrate the feasibility and efficiency of the proposed approach in one of typical application: image/video retrieval. In order to testify the validity of the method presented in the paper, a great number of experiments on an image database are performed. The database holds 1700 color pictures, which is composed of flowers, vehicles, landscape, animal, food, sea beach, construction and etc. So that the variety of images in the test set prevented any bias toward particular type of images. The subdivision of the image used in our experience is four windows. To evaluate the test results we use the values precision rate and recall rate are defined as follows

$$
\begin{aligned}
& \text { precision }=\frac{\text { number of relevant images selected }}{\text { total number of retrieved images }} \\
& \text { recall }=\frac{\text { numberof relevant images selected }}{\text { totalnumberof similarimagesin the database }}
\end{aligned}
$$

Fig.4 presents the average precision curves of different representations and similarity measure without any indexing scheme. The performances of the proposed method using local histograms with permuto-metric similarity measure are evaluated and compared with the local histograms and the global color histogram on the classic similarity $L_{1}$. From Fig.4, it is clear that the proposed approaches using local histograms performed better then the global histogram specific image representation based on the average precision. Whereas, the performances of permuto-metric measure are slightly better among all approaches.

Fig.5 shows retrieval results using the same images color. We compared our method with traditional approaches combined with the distance $L_{1}$. Our method can return more correctly retrieved images than traditional methods.

\section{CONCLUSION}

This paper has shown that the local histograms using the Minkowski distance $\left(L_{p}\right)$ (for example $\mathrm{p}=1$ ) in similarity measurement phase do not provide good performance. The reason is that a transformation of visual content of the image by rotation or translation does not change the degree of similarity. The pemuto-metric measure is proposed to solve this problem. Also, the experiments demonstrate that the pemuto-metric measure provides better performance than the classic distance $L_{1}$.

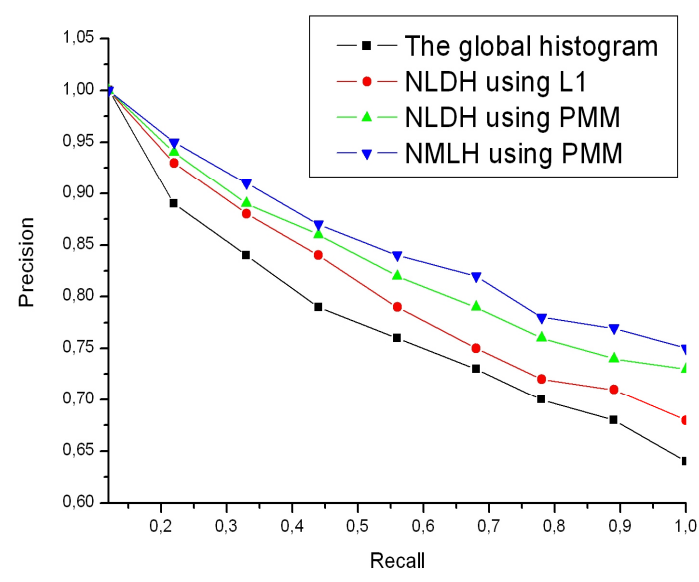

Fig. 4: Average recall-precision graph for the retrieval within the key-frames image database, using RGB color representation. 


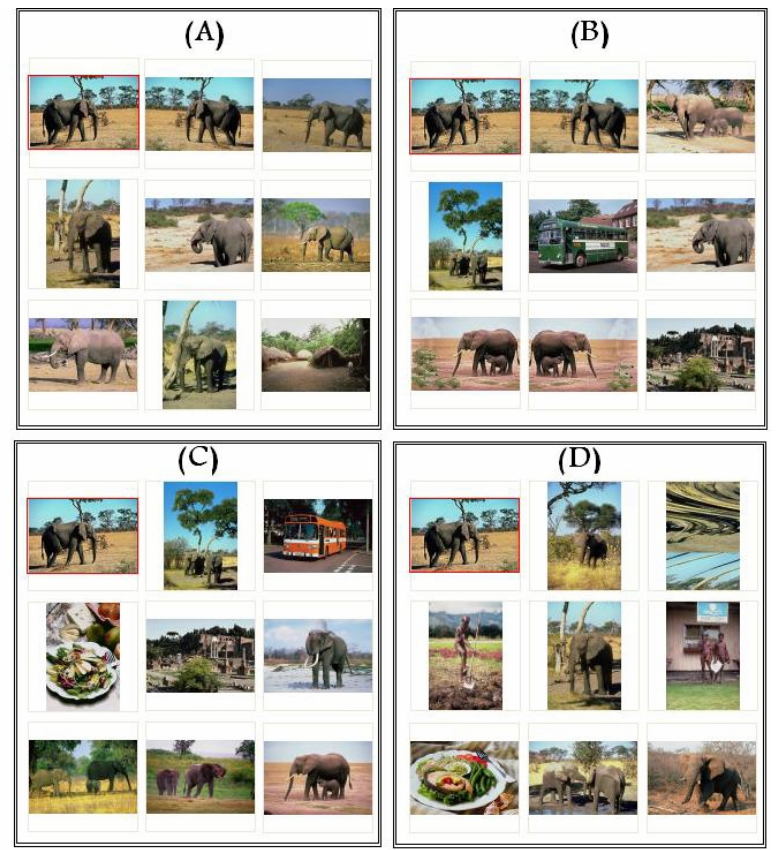

Fig. 5: Query images: (A) NMLH using PMM, provide a $88 \%$ accuracy. (B) NLDH using PMM, provide a $78 \%$ accuracy, (C) NLDH using $L_{1}$, provide a $66 \%$ accuracy. (D) Global histogram, provide a $56 \%$ accuracy.

\section{REFERENCES}

[1] M.Swain and D.Ballard, "Color indexing, "International Journal of Computer Vision, 7(1): 11-32, 1991., 1991.

http://www.springerlink.com/content/n231141541p1211g/

[2] J. Huang, S. Kumar, M. Mitra, W.J. Zhu, and R. Zabih. "Image indexing using color correlograms, ” In Proc. IEEE Comp. Soc. Conf. Comp. Vis. and Patt. Rec., pages762--768, 1997. DOI: 10.1109/ACV.1996.572008

[3] G. Pass R.Zabih, " Histogram refinement for content-based image retrieval" IEEE Workshop on Applications of computer Vision, pp. 96-102, 1996 DOI: 10.1109/ACV.1996.572008

[4] N. Boujemaa, S. Boughorbel, C. Vertan. Soft Color Signatures for Image Retrieval by Content, in: Eusflat'2001, 2001, vol. 2, p. 394-401. http://www.eusflat.org/publications/proceedings/EUSFLAT_2001/pa pers/394_Boujemaa.pdf

[5] M.W. Richardson, "Multidimensional Psychophysics," Psychological Bull., vol. 35, pp. 639-660, 1938.

[6] R.C. Veltkamp, "Shape matching: Similarity measures and algorithms", in Proceedings of the International Conference on Shape Modeling and Applications, pp. 188-197, May 2001. http://www.cs.uu.nl/research/techreps/repo/CS-2001/2001-03.pdf

[7] Rao, A.B., Srihari, R.K., Zhang, Z.F., 1999. Spatial color histogram for content-based retrieval. Tools with artificial intelligence. In: Proceedings of 11th IEEE International Conference. pp. 183-186. http://portal.acm.org/citation.cfm?id=853673

[8] C.C. Aggarwal, A. Hinneburg, and D.A. Keim, "On the Surprising Behavior of Distance Metrics in High Dimensional Space, ” Proc. Int'l Conf. Database Theory, pp. 420-434, 2001. DOI 10.1007/3-540-44503-X_27

[9] A. Sturn, "Cluster Analysis for Large Scale Gene Expression Studies", Master Thesis, Graz University of Technology, Graz, Austria, 2001. http://genome.tugraz.at/Theses/Sturn2001.pdf

[10] Sarfraz, M. Ridha, A."Content-based Image Retrieval using Multiple Shape Descriptors"Computer Systems and Applications, AICCSA '07. IEEE/ACS International Conference pages 730-737, 2007.

[11] T. Chang and C. kuo, "Texture analysis and classification using tree structured wavelet transform, " IEEE Trans. Image Processing, vol. 4, pp. 863-870, 1993.

[12] J. Malik and P. Perona, "Preattentive texture dissimilarity with early vision mechanisms, " J. Optical Soc. Am. A, vol. 7, no. 5, pp. 923-932, 1990.
[13] E. Hadidemetriou, M. D. Grossberg, and S.K. Nayar, "Multiresolution histograms and their use for recognition, " IEEE Transactions of Pattern Analysis and Machine Intelligence, vol. 26, no. 7, pp. 831-847, July 2004.

[14] A. Bovik, M. Clark, and W. Geisler, "Multichannel texture analysis using localized spatial filters, " IEEE Transactions of Pattern Analysis and Machine Intelligence, vol. 12, no. 1, pp. 55-73, Jan. 1990.

[15] J. Engel, "The multiresolution histogram, " Metrika, vol. 46, pp. 41-57, 1997.

http://www.springerlink.com/content/w27314v9417642u5/

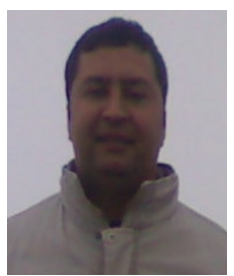

Alaoui Rachid was born in morocco in 1977. Received the master's degrees in Applied Mathematics from UCAM-Marrakech University in 2001. He is currently working toward the $\mathrm{PhD}$ degree in the LISQ Laboratory (Laboratory of Computer Science, Quality and Statistics) at USMBA-Fez University. His research area of interests are Image Processing and Pattern Recognition. 